\title{
Acute Pancreatitis with Normal Amylase and Lipase
}

\author{
Kichul Ko, MD, Luz Catherine Tello, MD, Jessica Salt, MD
}

\section{Introduction}

Acute pancreatitis is diagnosed by clinical history and physical examination with concurrent elevations in serum amylase and lipase levels; occasionally radiographic findings are of further assistance. We report a case of radiographically proven acute pancreatitis with normal serum amylase and lipase levels.

\section{Case Report}

A 35 year old male patient was in his usual state of health until four days prior to presentation when he began experiencing severe epigastric abdominal pain accompanied by nausea and vomiting. On Day 2 of his symptoms, he reported to the emergency department and was discharged home with a diagnosis of viral gastroenteritis. His symptoms worsened causing his return to the emergency department the following day for re-evaluation. The patient's past medical history included recently diagnosed diabetes mellitus type 2 and gastroesophageal reflux disease (GERD). His medications included metformin and esomeprazole which he took for the first time on Day 1 of symptom onset and did not continue taking secondary to severe nausea and vomiting. He had no known drug allergies. He reported occasional use of alcohol with the last use about two months prior to this presentation. He smoked one and a half packs of cigarettes per day for 14 years. He denied any past or present illicit substance use. He reported no personal or family history of pancreatitis.

His admission laboratory findings were: WBC count $19.8 \mathrm{x}$ $10^{9} \mathrm{~B} / \mathrm{L}$ with $11 \%$ bands, hemoglobin $13.3 \mathrm{~g} / \mathrm{dL}$, platelets 282 x $10^{9} / \mathrm{L}$, creatinine $1.1 \mathrm{mg} / \mathrm{dL}$, aspartate aminotransferase 16 $\mathrm{IU} / \mathrm{L}$, alanine aminotransferase $24 \mathrm{IU} / \mathrm{L}$, alkaline phosphatase $78 \mathrm{IU} / \mathrm{L}$, lactate $23.1 \mathrm{mg} / \mathrm{dL}$, triglyceride $1195 \mathrm{mg} / \mathrm{dL}$, amylase $17 \mathrm{IU} / \mathrm{L}$ (normal <132) and lipase $25 \mathrm{IU} / \mathrm{dL}$ (normal <52). A CT scan of the abdomen revealed diffuse fatty liver infiltration, homogenously enhancing pancreas and significant peripancreatic infiltration with surrounding fluid consistent with a diagnosis of acute pancreatitis. It also showed gallstones with no ductal dilation. The abdominal ultrasound showed cholelithiasis with no evidence of cholecystitis.

Based on the clinical presentation and radiographic findings, the patient was diagnosed with acute pancreatitis secondary to hypertriglyceridemia. He was treated with bowel rest, hydration, and pain control. The patient was also found to have pneumonia which was treated with moxifloxacin. Gemfibrozil and simvastatin were added for his dyslipidemia. His symptoms continued to improve during the hospital course and he was discharged on Day 7 with medications for pain control, dyslipidemia, diabetes, GERD and pneumonia.

\section{Discussion}

The diagnosis of acute pancreatitis is made based on relevant clinical features including severe abdominal pain, nausea, vomiting and elevation of pancreatic enzymes with serum amylase and/or lipase levels usually three times the upper limit of normal. ${ }^{1,2}$ Abdominal imaging with CT, magnetic resonance imaging (MRI), or transabdominal ultrasonograph (US) can be helpful in confirming the diagnosis of pancreatitis or ruling out other etiologies of acute abdominal pain that may cause mild elevations of serum pancreatic enzymes ${ }^{1}$.

Lipase is a $48 \mathrm{kD}$ pancreatic enzyme that is involved in digestion. Lipase level increases within four to eight hours of acute pancreatitis, peaks at 24 hours, and remains elevated for one to two weeks, typically longer than amylase levels ${ }^{1,3,4}$. Lipase is more sensitive and specific than amylase in diagnosing acute pancreatitis, with a negative predictive value of $94 \%$ to $100 \%{ }^{3,4}$. Therefore, normal lipase level in the setting of acute abdominal pain is often used to rule out a diagnosis of acute pancreatitis. Our patient was admitted with a clinical picture and radiographic findings consistent with acute pancreatitis despite normal lipase and amylase levels throughout the hospital course. In the case of our patient, the etiology of acute pancreatis was hypertriglyceridemia. Previous studies suggested that the hyperlipemic serum may interfere with the amylase assay in vitro leading to false negative results. ${ }^{5}$ However, there have been no previous reports on the effect of serum triglyceride on lipase level analysis. A literature search yielded two cases of symptomatic acute pancreatitis with normal lipase levels similar to our patient, however, neither reported the likely etiology for acute pancreatitis ${ }^{3,4}$. While it is possible that this presentation was an acute-on-chronic attack secondary to longstanding pancreatitis caused by hypertriglyceridemia, this is an unlikely explanation based on clinical and radiographic findings. This was the first reported symptomatic occurrence of pancreatitis in this patient and the radiographic findings did not reveal calcifications or fibrosis in the pancreas that is commonly associated with chronic pancreatitis.

This is an unusual case of acute pancreatitis with normal amylase and lipase levels in the setting of hypertriglyceridemia. This case highlights that while laboratory findings are useful diagnostic criteria, the absence of such findings should not replace clinical judgment used in formulating most appropriate diagnosis.

\section{References}

1. Swaroop VS, Chari ST, Clain JE. Severe Acute Pancreatitis. JAMA. 2004; 291:2865-8.

2. Whitcomb DC. Acute Pancreatitis. N Engl J Med. 2006; 354:2142-50.

3. Cartier T, Sogni P and Perruche F, et al. Normal lipase serum level in acute pancreatitis: a case report. Emerg Med J. 2006; 23:701-2.

4. Fan $\mathrm{H}$, Chen $\mathrm{A}$, Zhang $\mathrm{X}$, et al. Severe acute pancreatitis with normal lipase serum level complicating leukemoid reaction. J Chin Clin Med. 2009; 473-5.

5. Fallat RW, Vester JW, Glueck CJ. Suppression of Amylase Activity by Hypertriglyceridemia. JAMA. 1973; 225:1331-4. 